\title{
Alpers-Huttenlocher syndrome
}

INSERM

\section{Source}

INSERM. (1999). Orphanet: an online rare disease and orphan drug data base. AlpersHuttenlocher syndrome. ORPHA:726

Alpers Huttenlocher syndrome (AHS) is a cerebrohepatopathy and a rare and severe form of mitochondrial DNA (mtDNA) depletion syndrome characterized by the triad of progressive developmental regression, intractable seizures, and hepatic failure. 